FACTORES DE RIESGO PARA BACTERIEMIA EN NEUTROPENIA FEBRIL EN PACIENTES PEDIÁTRICOS HEMATO-ONCOLÓGICOS RISK FACTORS FOR BACTEREMIA IN PEDIATRIC HEMATO-ONCOLOGY PATIENTS WITH FEBRILE NEUTROPENIA

Melissa López Víquez Kattia Camacho Badilla² Kathia Valverde Muñoz ${ }^{3}$

1,2 y 3 Médico pediatra. Caja costarricense del seguro social, San José Costa Rica.

Contacto: melilov@gmail.com

\title{
RESUMEN
}

Objetivo:

La neutropenia febril es una complicación frecuente en pacientes hemato-oncológicos al recibir quimioterapia, con una morbi-mortalidad importante. En América Latina son escasos los estudios validando parámetros clínicos y de laboratorio que puedan utilizarse para clasificar el riesgo de bacteriemia, asociado a neutropenia febril. Métodos: Estudio retrospectivo, observacional. Se incluyeron los pacientes hemato-oncológicos hospitalizados por presentar neutropenia febril secundaria a quimioterapia en el hospital pediátrico del 1ro de enero 2016 al 31 de julio 2017. Resultados: Se incluyeron 318 episodios de neutropenia febril secundario a quimioterapia presentándose en 170 pacientes con cáncer durante 19 meses del estudio. Los episodios se dieron predominantemente en pacientes con leucemia linfocítica aguda (67\%); $77 \%$ de los episodios clasificándose como alto riesgo para bacteriemia

\section{Cómo citar:}

López Víquez, M., Camacho Badilla, K., \& Valverde Muñoz, $\mathrm{K}$. (2021). Factores de riesgo para bacteriemia en neutropenia febril en pacientes pediátricos Hemato-Oncológicos. Revista Ciencia Y Salud, 5(1), Pág. 4-12. después del análisis respectivo. En $2.2 \%$ de los casos se obtuvo identificación microbiológica en hemocultivos, prevaleciendo la infección por Gram negativos (62,5\%). No hubo mortalidad asociada. La presencia de hipotensión (OR: 29.9), catéter venoso central (OR: 8.1) y conteo plaquetario $\leq 50.000 / \mathrm{mm} 3$ (OR: 5.0) al ingreso fueron parámetros que confirieron mayor riesgo de bacteriemia en nuestra población. Conclusión: Con estos resultados, se han validado parámetros clínicos y de laboratorio que pueden aplicarse en la población costarricense para clasificar el riesgo de bacteriemia en los pacientes con neutropenia y fiebre. Así, optimizar el abordaje, adecuar la terapia antimicrobiana empírica, reducir el riesgo de resistencia antimicrobiana y mejorar la calidad de atención de los pacientes con cáncer en aras de mejorar la sobrevida de esta población.

Recibido: $27 /$ nov/2020

Aceptado: 01/feb/2021

Publicado: 15/feb/2021

Palabras Clave: pediatría, neutropenia febril, quimioterapia, factores de riesgos. 


\section{CIENCIA\&SALUD}

\section{ABSTRACT}

Aim: Febrile neutropenia is a frequent complication, with high mortality, of hemato/ oncology patients receiving chemotherapy. In Latinamerica there are few studies showning clinical or laboratory criteria to classify the patients in high risk for bacteremia, associated to fever with neutropenia. Methods: This is a retrospective, observational study. All hemato-oncology patients admitted at the Hospital pediatric, from January 1st, 2016 to July 31, 2017 with febrile neutropenia due to chemotherapy were included. Results: 318 episodes of fever and neutropenia due to chemotherapy were included of a total of 170 patients with cancer during the 19 months of the study. $77 \%$ of the episodes were classified as high risk for severe sepsis for bacteremia after analysis. 2.2\% had positive blood culture with a prevalence of Gram's negative bacteria (62,5\%). There were no deaths. The study population demonstrates higher risk for bacteremia on patients presenting hypotension (OR: 29.9), a central catheter (OR: 8.1) and platelets count of $\leq 50000 / \mathrm{mm} 3$ (OR: 5.0). Conclusion: With these results, it has been validated clinical and laboratory parameters that can be applied to the costarican population, to stratify the risk for bacteremia of patients with fever and neutropenia. This, in the future, optimizing the assessment and the appropriate empiric antimicrobial coverage, reducing the antimicrobial resistance and improving the outcome of complications of patients with cancer.

Keywords: Pediatrics, febrile neutropenia, chemotherapy, risk factors.

La neutropenia febril (NF) es una complicación frecuente en los pacientes hemato-oncológicos al recibir quimioterapia.1,2 Según la literatura mundial, se estima que el 10-50\% de los pacientes con tumores sólidos y más del $80 \%$ de las malignidades sanguíneas presentan como complicación la neutropenia febril.3,4 Estos inciden en mayores costos económicos hospitalarios y requieren de un internamiento promedio de 5-10 días;5-6 sin embargo un 45\% de los pacientes van a requerir una hospitalización prolongada.5

Se han publicado diferentes clasificaciones de riesgo a nivel internacional, con distintos parámetros clínicos y de laboratorio, que evalúan el riesgo de infección invasora severa. En Latinoamérica, en el 2001 Santolaya, et. al, publica una clasificación de riesgo para infección invasora donde describe que dentro de los factores para determinar que el paciente tiene riesgo alto para infección invasora incluyen: valores de proteína C-reactiva sérica $\geq 90 \mathrm{mg} / \mathrm{dl}$, presencia de hipotensión, recaída de leucemia como tipo de cáncer, conteo plaquetario $\leq 50000 / \mathrm{mm} 3$ y quimioterapia reciente ( $\leq 7$ días). 7 Se consideró de riesgo bajo a todos aquellos pacientes que no cumplen con ningún criterio establecido anteriormente.7

El objetivo principal de este estudio es validar la escala de clasificación de riesgo para infección bacteriana invasora publicada por Santolaya, et. al, en el 2001, que se encuentra basada en parámetros clínicos y de laboratorio7 y aplicarla como escala de riesgo para bacteriemia en pacientes que ingresaron con neutropenia febril en el Hospital Pediátrico.

\section{MÉTODOS}

Estudio retrospectivo, observacional donde se incluyeron todos los pacientes hemato-oncológicos con episodios de neutropenia y fiebre, que acudieron al hospital, del 1ro de enero 2016 al 31 de julio 2017. Estos pacientes fueron inicialmente evaluados en los servicios de urgencias y a nivel de la consulta externa de Hemato-Oncología y posteriormente hospitalizados. En estos pacientes se analizaron parámetros clínicos y de laboratorio de la escala de clasificación de riesgo de Santolaya,7 con el objetivo de relacionar estas variables de riesgo para bacteriemia y su aplicación en la población pediátrica hemato-oncológica local.

Se ha definido neutropenia en el paciente oncológico como recuento de neutrófilos absolutos menor a 


\section{CIENCIA\&SALUD}

$500 / \mathrm{mm} 3$ o menor a 1000/mm3 si se espera una disminución a menos de 500/mm3 en las siguientes 24$48 \mathrm{hrs} .8-9$ Fiebre se define como toma de temperatura axilar mayor a $38,5^{\circ} \mathrm{C}$ o dos tomas mayores a $38^{\circ} \mathrm{C}$, separadas al menos por una hora.10,11 Bacteriemia fue definida como uno o más hemocultivos positivos por patógenos bacterianos, exceptuando los Staphylococcus coagulasa negativos, para los cuales se requerían 2 o más hemocultivos positivos.7 Para el momento del estudio, aún no se contaba con métodos moleculares de análisis que sirvieran como herramienta para optimizar la identificación microbiológica. Entre las variables cualitativas analizadas están: edad del paciente, tipo de malignidad, estadío de la enfermedad, tipo de quimioterapia. Con respecto a las variables cuantitativas están: fiebre, presión arterial, pruebas de laboratorio (proteína C-reactiva y hemograma), entre otras.

Se realizó análisis univariado con las variables categóricas determinadas en la escala como factores de riesgo con respecto a hemocultivos positivos y a cambios de antimicrobiano, usándose un nivel de significancia de $<0.05$ y su respectivo intervalo de confianza de 95\%. Se realiza el test exacto de Fisher para la variable de conteo plaquetario. Se realizó el análisis estadístico con los programas de cómputo: Epiinfo versión 7 , Excel 2007 y STATA versión 14. Este estudio fue debidamente aprobado por el Comité Ético Científico del HNN, San José, Costa Rica con el número de protocolo CEC-HNN-011-2017.

\section{RESULTADOS}

Un total de 318 episodios de NF se presentaron en 170 pacientes hemato-oncológicos. La caracterización de dicha población se presenta en el cuadro 1.

Los pacientes con el diagnóstico de leucemia linfocítica aguda (LLA) fueron los que más episodios de NF presentaron (67,0\%), seguidos por pacientes con tumores sólidos $(24,2 \%)$ y luego linfomas $(7,9 \%)$. Al clasificar el riesgo para infección bacteriana invasora, según los criterios publicados por Santolaya en su estudio 2001,7 se obtuvo que de los 318 casos incluidos, 245 episodios (77\%) de NF fueron clasificados como alto riesgo para infección invasora bacteriana.

Hubo 14 casos con hemocultivos positivos; sin embargo, siete de ellos se consideraron contaminantes. De los casos de bacteriemias, un 62.5\% correspondió a bacterias Gram negativas, siendo las más frecuentes: Klebsiella pneumoniae (40\%), Pseudomonas aeruginosa (40\%), y Escherichia coli (20\%). No se aisló ningún hongo o micobacteria. Se demostró asociación para obtener hemocultivos positivos en las siguientes variables: presencia de hipotensión (OR: $29.9,95 \% \mathrm{Cl} 2.12-263.60$ ), presencia de un catéter venoso central (OR: $8,95 \% \mathrm{Cl} 1.3-56.3$ ), conteo de plaquetas $\leq 50.000 / \mathrm{mm} 3$ (OR: $4.9 ; 95 \% \mathrm{Cl} 1.0-22.8$ ) y PCR menor o igual a $90 \mathrm{mg} / \mathrm{L}$ (OR: 0.14; 95\% Cl 1.1-0.9) A los 78 casos a quienes se les realizó PCT, todos obtuvieron hemocultivos negativos.

Adicionalmente, un $63 \%$ de nuestra población de neutropenia febril, ameritó escalonamiento de la terapia antimicrobiana por parte del equipo tratante, ya sea por persistencia de la fiebre, deterioro clínico asociado o por la presencia de neutropenia profunda. Las variables que mostraron significancia estadística para cambio de antimicrobiano fueron: tipo de malignidad, tiempo posterior a quimioterapia menor a 7 días (OR, 1.65; $95 \% \mathrm{Cl}, 1.01-2.69$ ), conteo de neutrófilos absolutos menor a $100 / \mathrm{mm} 3$ (OR, $2.13 ; 95 \% \mathrm{Cl}, 1.30-3.52$ ) y conteo plaquetas menor a $50000 / \mathrm{mm} 3(\mathrm{OR}, 2.26 ; 95 \% \mathrm{Cl}, 1.27-4.04)$ al ingreso.

Ninguno de los pacientes del estudio que ingresó por fiebre falleció durante el internamiento

\section{DISCUSIÓN}

La población incluida durante el período del estudio constituyó todo el universo de los pacientes hemato-oncológicos con NF, ya que el hospital es el único centro de referencia nacional para manejo de estas patologías. Dentro de las características de la población la LLA es la malignidad más frecuente que presenta neutropenia febril como complicación, como se ha descrito en la literatura internacional y esto se debe a que la LLA es la neoplasia de mayor incidencia no solo en nuestro país, sino también a nivel mundial. 7, 12-15 


\section{CIENCIA\&SALUD}

A nivel mundial, se reporta que el $20-24 \%$ de los casos de neutropenia febril secundaria a quimioterapia tienen hemocultivos positivos.16-18 En nuestra población se logró la identificación microbiológica en un $13.5 \%$, y solo en un $2.2 \%$ se obtuvo un hemocultivo positivo. Este dato fundamenta el hecho de revisar la calidad de la muestra del hemocultivo y ampliar metodologías moleculares en la identificación de los gérmenes. Otras publicaciones han demostrado que dentro de los aislamientos por bacterias Gram negativas, los de mayor frecuencia en orden descendente son Klebsiella pneumoniae, Escherichia coli y Pseudomonas aeruginosa,18-19 tal como se reporta en nuestros resultados. 15

En la literatura se han validado múltiples parámetros que se presentan como factores de riesgo para complicaciones, sepsis severa o incluso mortalidad en la población descrita.3,7,12,14,20-25 En la población estudiada, la presencia de hipotensión, presencia de cáteter venoso central y trombocitopenia menor o igual a 50,000/mm3, muestran mayor riesgo para la presencia de hemocultivos positivos. En cuanto al análisis de los parámetros inflamatorios, se confirma lo demostrado en la literatura7,20, donde el valor de PCR mayor o igual a $90 \mathrm{mg} / \mathrm{L}$, está relacionado con la presencia de hemocultivos positivos. Por otro lado, en los pacientes a quienes se les realizó PCT, y que tuvieron valores por encima de $0,5 \mathrm{ng} / \mathrm{mL}$, ninguno presentó hemocultivo positivo. Según la literatura, la PCT es un marcador que requiere más estudios comparativos en esta población para definir su validez como valor predictivo en el manejo de NF.19, 26-29

Cabe mencionar que a pesar de que el $77 \%$ de los pacientes de nuestro estudio fueron clasificados como alto riesgo para bacteriemia según criterios de Santolaya et. al,7 en la población analizada no se presentó ningún fallecimiento.

Durante el período del estudio, el protocolo a nivel local para manejo de los pacientes con NF incluía la administración de Clindamicina más un aminoglicósido como terapia empírica. En muchos de los pacientes hospitalizados por neutropenia febril, durante la admisión fue necesario realizar escalonamiento de la terapia antimicrobiana en el $63 \%$ de los pacientes, basado sobre todo en el criterio clínico del médico tratante, y en la mayoría de los casos sin tener confirmación de agentes infecciosos; por lo que se mostró una necesidad de realizar una revisión del protocolo local para manejo de esta población.

Dentro de las limitaciones del estudio, se tuvo que no se hizo análisis individual por patología oncológica, siendo la mayoría leucemias linfocíticas agudas. La baja positividad de los hemocultivos demuestra que debe haber una mejoría en este aspecto. La principal fortaleza es el análisis reciente de la población pediátrica oncológica nacional.

Uno de los alcances que permitió este estudio fue la creación de una nueva guía de manejo de los pacientes hemato-oncológicos que ingresan con neutropenia febril al HNN, con base en la revisión de los gérmenes que se encontraron como etiología en los episodios de NF presentada en este estudio. Se analizó la necesidad de modificar la terapia antibiótica empírica, cambiándose a Piperazilina-Tazobactam en pacientes de bajo riesgo y en pacientes de alto riesgo, Ceftazidime más Amikacina.

Adicionalmente, este estudio mostró la necesidad de analizar aspectos relacionados con las muestras de los hemocultivos, recalcando la importancia en el volumen de sangre a introducir en cada botella, de acuerdo con el peso del paciente y siguiendo las recomendaciones del fabricante. Esto con la finalidad de mejorar la positividad de los hemocultivos en esta población.

Finalmente, nuestro análisis permite demostrar que algunos de los criterios de la escala de clasificación de riesgo publicada por Santolaya et al, es aplicable a la población pediátrica de nuestro país, y podría ser un instrumento útil para otras poblaciones pediátricas latinoamericanas. 


\section{CIENCIA\&SALUD}

\section{REFERENCIAS BIBLIOGRÁFICAS}

1. Davis K, Wilson S. Febrile neutropenia in paediatric oncology. Paediatr Child Health (Oxford). 2020; 30(3):93-97

2. Paolino J, Mariani J, Lucas A, et al. Outcomes of a clinical pathway for primary outpatient management of pediatric patients with low-risk febrile neutropenia. Pediatr Blood Cancer. 2019; 66(7)e27679.

3. Klastersky J. Management of Fever in Neutropenic Patients with Different Risks of Complications. Clin Infect Dis. 2004;39 (Suppl 1): S32-S37.

4. Mohammed H, Yismaw M, Fentie A, et al. Febrile neutropenia management in pediatric cancer patients at Ethiopian Tertiary Care Teaching Hospital. BMC Res Notes 2019; 12, 528

5. Basu S, Fernandez I, Fisher S, Asselin B, Lyman G. Length of Stay and Mortality Associated with Febrile Neutropenia Among Children With Cancer. J ClinOncol. 2005; 23:7958-7966.

6. Pérez-Heras Í, et al. Neutropenia febril posquimioterapia. Estancia hospitalaria y experienciaen nuestro medio. An Pediatr (Barc). 2019; 92(3): 141-146

7. Santolaya M, Alvarez A, Becker A, et al. Prospective, multicenter evaluation of risk factors associated with invasive bacterial infection in children with cancer, neutropenia, and fever. J. ClinOncol2001; 19: 3415-21.

8. Keng M, Sekeres M, Febrile Neutropenia in Hematologic Malignancies. CurrHematolMaligRep 2013; 8(4): 370-378.

9. Kebudi R, Kizilocak H. Febrile Neutropenia in Children with Cancer: Approach to Diagnosis and Treatment. CurrPediatr Rev. 2018;14(3):204-209.

10. Ducasse K, Fernández J, Salgado C, Álvarez A, Avilés C, Becke A, et al. Caracterización de los epi sodios de neutropenia febril en niños con leucemia mieloide aguda y leucemia linfoblástica aguda. RevChilenalnfectol 2014; 31 (3): 333-338.

11. Rivera-Salgado D, Valverde-Muñoz K, Ávila-Agüero M. Febrile neutropenia in cancer patients: management in the emergency room. RevChilenalnfectol. 2018;35(1):62-71

12. Avilés-Robles M, Ojha R, González M, Ojeda-DiezbarrosoK, Dorantes-Acosta E, Jackson B, et al. Bloodstream infections and inpatient length of stay among pediatric cancer patients with febrile neutropenia in Mexico City. American Journal of Infection Control. 2014; 42: 1235-7

13. Miedema K, Winter R, Ammann R, Droz S, Spanjaard L, Bont E, et al. Bacteria causing bacteremia in pediatric cancer patients presenting with febrile neutropenia-species distribution and susceptibility patterns. Support Care cancer. 2013; 21 (9): 2417-2426.

14. Paganini H, Aguirre C, Puppa G; et al. A Prospective, Multicentric Scoring System to Predict Mortality in Febrile Neutropenic Children With Cancer. Cancer. 2007; 109 (12): 2572-79

15. Ojha R, Asdahl P, Steyerberg E, Schroeder H. Predicting bacterial infections among pediatric cancer patients with febrile neutropenia: External validation of the PICNICC model. Pediatr Blood Cancer. 2017; 65(4)e26935

16. Kara S, Tezer H, Polat M, et al. Risk factors for bacteremia in children with febrile neutropenia. Turk J Med Sci. 2019; 49(4):1198-1205

17. Petty L, Sokol E, Bartlett A, et al. Repeated Blood Cultures in Pediatric Febrile Neutropenia: Would Following the Guidelines Alter the Outcome? Pediatr Blood Cancer. 2016; 63:1244-1249

18. Escrihuela-Vidal F, Laporte J, Albasanz-Puig A, Gudiol C. Update on the management of febrile neutropenia in hematologic patients. Rev EspQuimioter. 2019; 32(Suppl 2): 55-58.

19. Hemming $\vee$, Jakes A, Shenton G, Phillips B. Prospective cohort study of procalcitonin levels in children with cancer presenting with febrile neutropenia. BMC Pediatrics. 2017; 17: 2.

20. Lehrnbecher T, Phillips R, Alexander S, Alvaro F, Carlesse F, Fishe B, et al. American Society of Clinical Oncology Endorsement: Guideline for the Management of Fever and Neutropenia in Children with Cancer and/or Undergoing Hematopoietic Stem Cell Transplantation. J ClinOncol. 2012; 30: 4427-4438.

21. Phillips R, Lehrnbecher T, Alexander S, Sung L. Updated Systematic Review and Meta-Analysis of the Performance of Risk Prediction Rules in Children and Young People with Febrile Neutropenia. PLoS one. 2012; 7 (5): 1-9 
22. Badiei Z, Khalesi M, Alami M, et al. Risk factors associated with life-threatening infections in children with febrile neutropenia: a data mining approach.J Pediatr Hematol Oncol. 2011; 33(1)e9-

23. Hurtado I, Sánchez D, Espinal D, Garcé C. Evolución clínica y de laboratorio de episodios de neutropenia febril en niños con cáncer, en un hospital de Colombia, período 2007-2009. RevChile nalnfectol. 2012; 29 (6): 672-676.

24. Fletcher M, Hodgkiss H, Zhang S, Browning R, Hadden C, Hoffman C, et al. Prompt administration of antibiotics is associated with improved outcomes in febrile neutropenia in children with cancer. Pediatr Blood Cancer. 2013; 60:1299-1306

25. Jiménez E, Sackmann F, Garay G, Svarch E, Odio C, Bustelo P, et al. Tratamiento de la leucemia lin focítica aguda (LLA) en niños. Rev. Med. Hosp. Nal. Niños Costa Rica. 1985; 20 (2): 175-188

26. Alali M, David M, Danziger-Isakov L, et al. Pediatric Febrile Neutropenia Change in Etiology of Bacteremia, Empiric Choice of Therapy and Clinical Outcome. J PediatrHematol Oncol: May 12, 2020 - Volume Publish Ahead of Print issue

27. Santolaya M, Alvarez A, Aviles C, et al. Predictors of severe sepsis not clinically apparent during the first twenty-four hours of hospitalization in children with cancer, neutropenia, and fever.

Pediatr Infect Dis. 2008; 27: 538-43

28. Feld R. Bloodstream infections in cancer patients with febrile neutropenia. Int J Antimicrob Agents. 2008;32 (1): S30-3

29. Van der Galiën HT, Loeffen EAH, Miedema KGE, Tissing WJE. Predictive value of PCT and IL-6 for bacterial infection in children with cancer and febrile neutropenia. Support Care Cancer. 2018;26(11):3819-3826

\section{Cuadro 1. Caracterización general de los pacientes con neutropenia febril de la población hemato-on- cológica del Hospital pediátrico}

$\mathrm{N}=318$

Edad, meses

\begin{tabular}{|l|l|}
\hline Mediana 3DS & 54.5335 .7 \\
\hline Rango & $4-155$ \\
\hline
\end{tabular}

Sexo, n (\%)

\begin{tabular}{l|l} 
Masculino & $184(57.9)$ \\
\hline Femenino & $134(42.1)$ \\
\hline
\end{tabular}

\begin{tabular}{|l|l|}
\hline Malignidad, $\mathrm{n}(\%)$ & $213(67.0)$ \\
\hline LLA & $3 \quad(0.9)$ \\
\hline LMA & $25 \quad(7.9)$ \\
\hline Linfomas & $77 \quad(24.2)$ \\
\hline Tumor sólido &
\end{tabular}

Recaída leucemias, n/N (\%)

\begin{tabular}{l|l} 
Sí & $37 / 213 \quad(17.4)$ \\
\hline No & $176 / 213 \quad$ (82.6) \\
\hline
\end{tabular}

Fase de quimioterapia, $\mathrm{n} / \mathrm{N}(\%)$

\begin{tabular}{l|ll}
\hline Inducción & $85 / 224 \quad$ (38.0) \\
\hline Consolidación/ intensificación & $31 / 224 \quad(13.8)$ \\
\hline Mantenimiento & $108 / 224 \quad(48.2)$ \\
\hline
\end{tabular}

Tiempo postquimioterapia, n (\%)

\begin{tabular}{|l|l}
\hline Igual o menor a 7días & $202(63.5)$ \\
\hline Mayor a 7 días & $116(36.5)$ \\
\hline
\end{tabular}

Mucositis, $\mathrm{n}(\%)$ 


\begin{tabular}{|c|c|}
\hline Sí & $44 \quad(13.9)$ \\
\hline No & $274(86.1)$ \\
\hline \multicolumn{2}{|l|}{ Hipotensión, n (\%) } \\
\hline Sí & $6 \quad(1.9)$ \\
\hline No & $312(98.1)$ \\
\hline \multicolumn{2}{|l|}{ Catéter central, n (\%) } \\
\hline Sí & $48 \quad(15.1)$ \\
\hline No & $270(84.9)$ \\
\hline \multicolumn{2}{|c|}{ Temperatura, n/N (\%) } \\
\hline $37.7-38.0^{\circ} \mathrm{C}$ & 116/297 (39.0) \\
\hline $38.1-39.0^{\circ} \mathrm{C}$ & $165 / 297(55.6)$ \\
\hline Mayor a $39.0^{\circ} \mathrm{C}$ & $16 / 297 \quad(3.4)$ \\
\hline \multicolumn{2}{|c|}{ Riesgo clasificado*, n (\%) } \\
\hline Bajo & $73 \quad(23.0)$ \\
\hline Alto & $245(77.0)$ \\
\hline
\end{tabular}




\begin{tabular}{|c|c|c|c|c|}
\hline & $\begin{array}{l}\text { Hemocultivo posi- } \\
\text { tivo }\end{array}$ & $\begin{array}{l}\text { Hemocultivo nega- } \\
\text { tivo }\end{array}$ & $\begin{array}{l}\text { Odds ratio }(95 \% \\
\mathrm{Cl})\end{array}$ & $P$ \\
\hline \multicolumn{5}{|l|}{ Malignidad } \\
\hline LLA & $5(2.44)$ & $200(97.56)$ & - & 0.869 \\
\hline LMA & $0 \quad(0.00)$ & $\begin{array}{ll}3 & (100.00) \\
\end{array}$ & - & \\
\hline Linfomas & $\begin{array}{ll}0 & (0.00)\end{array}$ & $25 \quad(100.00)$ & - & \\
\hline Tumor sólido & $2 \quad(2.60)$ & $75 \quad(97.40)$ & - & \\
\hline \multicolumn{5}{|l|}{ Recaída Leucemia } \\
\hline Sí & $1 \quad(2.70)$ & $36 \quad(97.30)$ & 1.14 & 0.904 \\
\hline No & $4 \quad(2.37)$ & $165(97.63)$ & $(0.22-12.03)$ & \\
\hline \multicolumn{5}{|c|}{ Tiempo postquimioterapia } \\
\hline $\begin{array}{l}\text { Igual o menor a } \\
\text { 7días }\end{array}$ & $6(3.03)$ & $192(96.97)$ & 3.47 & 0.224 \\
\hline Mayor a 7 días & $1 \quad(0.89)$ & $111 \quad(99.11)$ & $(0.41-160.94)$ & \\
\hline \multicolumn{5}{|l|}{ Hipotensión } \\
\hline Sí & $2(33.33)$ & $4 \quad(66.67)$ & 29.90 & menor que 0.00 \\
\hline No & $5 \quad(1.64)$ & $299(98.36)$ & $(2.12-263.60)$ & 1 \\
\hline \multicolumn{5}{|l|}{ Catéter central } \\
\hline Sí & $4(8.51)$ & $43 \quad(91.49)$ & 8.06 & 0.002 \\
\hline No & $3(1.14)$ & $260(98.86)$ & $(1.30-56.32)$ & \\
\hline \multicolumn{5}{|l|}{ Temperatura $\left({ }^{\circ} \mathrm{C}\right)$} \\
\hline $37.7-39.0$ & $6 \quad(2.20)$ & $267(97.80)$ & - & 0.305 \\
\hline Mayor a 39.0 & $1(6.25)$ & $15(93.75)$ & - & \\
\hline \multicolumn{5}{|l|}{ Neutrófilos (/mm3) } \\
\hline $\begin{array}{l}\text { Menor o igual a } \\
100\end{array}$ & $5(3.05)$ & $159(96.95)$ & 2.26 & 0.321 \\
\hline Mayor a 100 & $2(1.36)$ & $144(98.64)$ & $(0.36-24.09)$ & \\
\hline \multicolumn{5}{|l|}{ Plaquetas (/mm3) } \\
\hline $\begin{array}{l}\text { Menor o igual } \\
50000\end{array}$ & $4(5.88)$ & $64 \quad(94.12)$ & 4.98 & 0.0039 \\
\hline Mayor a 50000 & $3(1.24)$ & $239(98.76)$ & $(1.08-22.81)$ & \\
\hline \multicolumn{5}{|c|}{ Procalcitonina $(\mathrm{ng} / \mathrm{mL})$} \\
\hline $\begin{array}{l}\text { Menor o igual a } \\
0.5\end{array}$ & $0 \quad(0.00)$ & $58 \quad(100.00)$ & & \\
\hline Mayor a 0.5 & $0 \quad(0.00)$ & $20 \quad(100.00)$ & & \\
\hline \multicolumn{5}{|c|}{ Proteína C-reactiva $(\mathrm{mg} / \mathrm{L})$} \\
\hline Menor o igual a 90 & $2(0.95)$ & $209(99.05)$ & 0.14 & 0.008 \\
\hline Mayor a 90 & $5 \quad(6.33)$ & $74 \quad(93.67)$ & $(0.13-0.89)$ & \\
\hline
\end{tabular}




\begin{tabular}{|l|l|l|l|l|}
\hline \multicolumn{5}{|l|}{ Riesgo para infección severa ${ }^{*}$} \\
\hline Bajo & $1(1.43)$ & $69(98.57)$ & 0.56 & 0.595 \\
\hline Alto & $6(2.50)$ & $234(97.50)$ & $(0.01-4.78)$ & \\
\hline${ }^{\star}$ Según criterios de Santolaya, 2001.7 & \\
\hline
\end{tabular}

